\title{
LOX-1 ligands containing apolipoprotein B and carotid intima- media thickness in middle-aged community-dwelling US Caucasian and Japanese men
}

\author{
Tomonori Okamura $^{a, b}$, Akira Sekikawa $^{c}$, Tatsuya Sawamura ${ }^{d}$, Takashi Kadowaki ${ }^{b}$, Emma $^{2}$ \\ Barinas-Mitchellc ${ }^{\text {, Rachel H. Mackey }}{ }^{\mathrm{C}}$, Aya Kadota ${ }^{\mathrm{b}}$, Rhobert W. Evans ${ }^{\mathrm{c}}$, Daniel \\ Edmundowicz $^{\mathrm{e}}$, Aya Higashiyama ${ }^{\mathrm{f}}$, Yasuyuki Nakamurag, Robert D. Abbott ${ }^{\mathrm{h}}$, Katsuyuki \\ Miura $^{b}$, Akira Fujiyoshi ${ }^{b}$, Yoshiko Fujitad ${ }^{d}$, Yoshitaka Murakami', Naomi Miyamatsuj, Akemi \\ Kakino $^{d}$, Hiroshi Maegawa ${ }^{k}$, Kiyoshi Murata', Minoru Horie ${ }^{k}$, Kenichi Mitsunami ${ }^{m}$, Atsunori \\ Kashiwagi $^{k}$, Lewis H. Kuller ${ }^{c}$, and Hirotsugu Ueshima ${ }^{b}$ for the ERA JUMP Study Group \\ aPreventive Medicine and Public Health, School of Medicine, Keio University, Tokyo, Japan \\ bHealth Science, Shiga University of Medical Science, Otsu, Shiga, Japan \\ 'Epidemiology, University of Pittsburgh, Pittsburgh, Pennsylvania, USA \\ dVascular Physiology, National Cerebral and Cardiovascular Center, Suita, Osaka, Japan

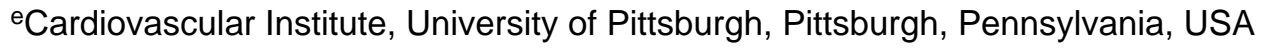 \\ fEnvironmental and Preventive Medicine, Hyogo College of Medicine, Nishinomiya, Hyogo, Japan \\ 'Cardiovascular epidemiology, Kyoto Women's University, Kyoto, Japan \\ hBiostatistics and epidemiology, University of Virginia, Charlottesville, Virginia, USA \\ 'Medical statistics, Shiga University of Medical Science, Otsu, Shiga, Japan \\ iClinical Nursing, Shiga University of Medical Science, Otsu, Shiga, Japan \\ kInternal Medicine, Shiga University of Medical Science, Otsu, Shiga, Japan \\ 'Radiology, Shiga University of Medical Science, Otsu, Shiga, Japan \\ mFamily medicine, Shiga University of Medical Science, Otsu, Shiga, Japan
}

\begin{abstract}
Objective-The serum level of LOX-1 ligand containing ApoB (LAB) may reflect atherogenicity better than LDL cholesterol (LDLC), total LDL particles and usual measurement of oxidized LDL. The association between LAB and intima-media thickness (IMT) of carotid artery was investigated by ultrasound in US and Japan men.
\end{abstract}

Methods-Participants were 297 US Caucasian and 310 Japanese men, aged 40 to 49 years without past history of cardiovascular disease. Serum LAB levels were measured by ELISAs with recombinant LOX-1 and monoclonal anti-apolipoprotein B antibody.

Address for Correspondence: Tomonori Okamura, Department of Preventive Medicine and Public Health, School of Medicine, Keio University, 35 Shinanomachi, Shinjuku-ku, Tokyo 160-8582, Japan, okamura@z6.keio.jp, Phone: +81-3-5363-3759, Fax:

+81-3-3359-3686.

Conflict of interest

Investigators of LDL-C Study Group received no remuneration for conducting this study. 
Results-Serum LAB levels [median (interquartile range), $\mu \mathrm{g} / \mathrm{L}$ ] were $1,321(936,1730)$ in US Caucasians and $940(688,1259)$ in Japanese. For Caucasian men, average IMT was higher in higher LAB quartile, which was $0.653,0.667,0.688$, and $0.702 \mathrm{~mm}$, respectively (p for trend= 0.02 ). Linear regression analysis showed serum $L A B$ was significantly associated with IMT after adjustment for LDLC or total LDL particles in addition to other traditional or novel risk factors for atherosclerosis such as C-reactive protein. However, there was no significant relationship between LAB and IMT in Japanese men.

Conclusion-Serum LAB, a new candidate biomarker for residual risk, was associated with an increased carotid IMT in US Caucasian men independently of various risk factors; however, ethnic difference should be clarified in the future.

\section{Keywords}

Lectin-like oxidized LDL receptor 1; LOX-1 ligand containing ApoB; population; risk factors; carotid intima-media thickness

\section{Introduction}

Previous studies have established high serum level of total cholesterol or low density lipoprotein cholesterol (LDLC) as a major risk factor for coronary artery disease (CAD). [14] However, unfortunately, many CAD events are not prevented by only LDLC lowering therapy. There remain residual risk factors to be examined beyond LDLC. [5] Oxidative modification of LDL, one of the most studied modifications of LDL leading to cholesterol accumulation in monocytes/macrophages, plays a key role in atherogenesis. [6] Lectin-like oxidized LDL receptor 1 (LOX-1) is the receptor for modified LDL identified in endothelial cells. [7] Initially, LOX-1 was identified as a receptor to recognize LDL with cupric ionmediated oxidization or acetylated; until now, it has been clarified that LOX-1 recognized many types of modified LDL. [8-11] Recently, an assay to measure the biological activity of apolipoprotein B (ApoB)-containing lipoprotein based on binding to LOX-1 was developed [12], which might reflect atherogenicity of whole modified LDL better than one specific antigenic determinant of oxidized LDL. [13,14]

However, to our knowledge, no previous reports have examined the impact of serum levels of LAB on the intima-media thickness (IMT) of carotid artery. Furthermore, there is no evidence concerning ethnic difference in serum LAB level and its association with IMT. Accordingly, we performed a comparative study of middle-aged Japanese and US Caucasian men who were randomly selected from each surveyed community.

\section{Methods}

\subsection{Study population}

Participants of the present study were from a cross-sectional study to compare subclinical atherosclerosis findings between US and Japanese men. Details of the study methods were described elsewhere. [15-18] A total of 623 Japanese and US Caucasian men aged 40-49 years without past history of cardiovascular disease (310 US Caucasian men from Allegheny County, Pennsylvania, US and 313 Japanese men from Kusatsu City, Shiga, Japan) were randomly selected from each community. Informed consent was obtained from all participants. Sixteen participants were excluded because of missing information. The remaining 607 participants (297 US Caucasian men and 310 Japanese men) were included in the current analysis. 


\subsection{Examination}

Venipuncture was performed early in the clinic visit after a 12-hour fast. Serum total cholesterol (TC), high-density-lipoprotein cholesterol (HDL-C), triglycerides (TG) and glucose were measured. [15-18] LDL-C was estimated by the Friedewald formula. [19] Creactive protein (CRP) was measured by an immunosorbent assay and total LDL particles were measured by nuclear magnetic resonance (NMR) spectroscopy. [16] Diabetes was defined as a fasting serum glucose $\geq 7.0 \mathrm{mmol} / \mathrm{L}(126 \mathrm{mg} / \mathrm{dl})$, the use of anti-diabetic agents, or both. Hypertension was defined as either systolic blood pressure $\geq 140 \mathrm{mmHg}$, diastolic blood pressure $\geq 90 \mathrm{mmHg}$ or the use of antihypertensive agents. Body mass index (BMI) was calculated as weight $(\mathrm{kg})$ divided by the square of height $(\mathrm{m})$. Drinking and smoking habits for each participant were assessed by a self-administered questionnaire. Current drinking was defined as those who consumed alcohol $\geq 2$ times per week.

\subsection{Measurement of LAB and sLOX-1}

LOX-1 expressed on a cell surface, is proteolytically cleaved by an undetermined enzymatic system at the membrane proximal extracellular domain, and releases into the blood stream as soluble form. [20] Accordingly, the soluble form of lectin-like oxidized LDL receptor 1 (sLOX-1) in serum was also measured in addition to LAB. The samples for LAB and sLOX-1 measurement were shipped on dry ice to Biomarker Science Co., Ltd., Kyoto, Japan. Recently, one of the authors (T.S.) have developed a unique ELISA assay to detect serum or plasma LAB, and this system can measure the levels of LAB in the serum, such as remnant lipoprotein and modified LDL including oxidized LDL. ${ }^{12}$ The precision (coefficient of variance (CV) was $11.1 \%$ in the intra-assay and $15.5 \%$ in the inter-assay $(n=6)$. The serum levels of sLOX-1 were measured by sandwich ELISA using two kinds of monoclonal antibody against the extracellular domain of LOX-1, that is, TS92 and a chicken monoclonal anti human LOX-1 antibody HUC5-40. [21] The precision (CV) was 6.4\% in the intra-assay and $8.7 \%$ in the inter-assay $(n=6)$.

\subsection{Intima-media thickness of the carotid artery}

The scanning protocol was previously described. [15-18] We applied continuous-qualityassessment programs developed by the laboratory to assure the scanning quality within and between sites. [22] Using a Toshiba 140A scanner equipped with a 7.5 MHz linear array transducer, detailed B-mode images of the right and left common carotid artery (CCA), common carotid bifurcation, and the internal carotid artery were obtained at both centers. Images from the following segments were digitized: near and far walls of the distal CCA (one centimeter proximal to the carotid bulb), far wall of the bulb, and first centimeter of the far wall of the internal carotid artery (a total of 4 locations per side). IMT measures were determined by electronically tracing the lumen-intima interface and the media-adventitia interface these segments. The mean of all average readings across the 8 locations comprised the average IMT. We used average IMT in our current study. Between sonographer and between reader intra-class correlation coefficients for IMT were $>0.85$ and $>0.90$, respectively. [22]

\subsection{Statistical analysis}

For comparison of risk factors across the LAB quartiles, analysis of variance for means or Chi square test for proportions was used. Linear regression analyses were used to examine the contribution of log-transformed serum LAB to IMT with adjustment for age and further adjustment for HDLC, TG, hypertension, diabetes, BMI, sLOX-1 (log-transformed), current smoking, drinking, lipid lowering medication and CRP (model 1). Further adjustment for LDLC in model 2 or total LDL particles in model 3 was performed. 
All confidence intervals were estimated at the $95 \%$ level and significance was set at a $\mathrm{P}$ value of $<0.05$. The IBM SPSS Statistics 19 (IBM Japan, Tokyo) software was used for analysis.

\section{Results}

Table 1 shows the comparison of risk characteristics between 297 US Caucasian men and 310 Japanese men including median and interquartile range of LAB. Medians of LAB $[\mu \mathrm{g} /$ L] were in US and 940 in Japan, of which geometric means were 1287 and 934, respectively. Medians of serum 1 sLOX-1 [ng/L] were 520 and 328, respectively. Both serum levels of LAB and sLOX-1 were significantly higher in Caucasian men than in Japanese men ( $\mathrm{P}<0.001$, respectively). Serum levels of TG, LDLC and total LDL particles were equivalent among two populations. Japanese men had a less favorable profile of some risk factors compared with US Caucasian men, including hypertension, diabetes and cigarette smoking. However, Japanese men were significantly less obese, had higher levels of HDLC and lower levels of CRP than Caucasian men. Japanese men had significantly lower average IMT compared with Caucasian men.

Mean values of average IMT are plotted against median values of LAB for each quartile (Figure S1). In US men and combined cohort, median values of LAB were positively associated with mean average IMT, whereas there is no positive association between LAB and average IMT in Japanese men.

Table 2 shows the means and prevalence of cardiovascular risk factors for US Caucasian men in each LAB quartile. Among the factors, serum levels of TG, LDLC, total LDL particles and CRP significantly increased with rising serum level of LAB while HDLC levels significantly decreased. The percent of men who were current smokers also significantly increased. The average IMT showed a positive graded relationship with LAB quartile.

For Japanese men, as indicated in Table 3, serum TG, LDLC, total LDL particles, BMI and sLOX-1 rose significantly with increasing LAB levels while the prevalence of current drinkers was lower in the higher LAB quartiles. Although mean values of average IMT among upper two quartiles of LAB were higher than those among lower two quartiles, there was no significant association between LAB and average IMT.

Table 4 shows the results of linear regression analysis. In age-adjusted model, LAB was associated with average IMT only in US men, not in Japanese men. After adjustment for age, HDLC, TG, hypertension, diabetes, BMI, sLOX-1(log-transformed), smoking, alcohol drinking, lipid lowering medication and CRP (model 1), LAB also showed significant positive association with average IMT in US men, not in Japanese men. Further adjustment for LDLC (model 2) or total LDL particles (model 3) did not alter above-mentioned results. When LDLC, total LDL particles and LAB were entered in one statistical model, LAB was still significantly associated with average IMT in US men (partial regression coefficient: 0.036, SE: 0.015, P= 0.018). Above-mentioned results were almost similar after exclusion for participants who were taking lipid lowering medication (data not shown).

\section{Discussion}

This is the first study to compare the serum level of LAB and sLOX-1 between US and Japanese population; both of them were significantly higher in US Caucasian men than in Japanese men. Furthermore, serum LAB levels had a significant positive association with carotid average IMT in US men independent of serum level of LDLC or total LDL particles. 
However, we did not observe significant relationship between LAB and carotid IMT in Japanese.

Some previous studies examined the relationship between modified LDL and carotid IMT or CAD. However, most studies were only assessed oxidized LDL based on one specific antigenic determinant. For example, Liu et al reported oxidized LDL was associated with carotid IMT in asymptomatic familial combined hyperlipidemia family members. [23] Yamazaki et al also indicated circulating malondialdehyde-modified LDL level, a kind of oxidized LDL, contributed the atherogenic process assessed by carotid IMT in familial combined hyperlipidemia patients. [14] A German study showed that elevated plasma levels of oxidized LDL were predictive of CAD in a nested-case control study [24]; however, in their further study with a prolonged follow-up period, oxidized LDL was not associated with an increased risk for CAD after adjustment for the ratio of total cholesterol to HDLC. [25] Furthermore, an US nested-case control study also suggested oxidized LDL was not an independent overall predictor of CAD after adjustment for other lipid markers. [26]

In the present study, LAB was significantly associated with average IMT even after adjustment for LDLC and HDLC in US men. Furthermore, this relationship was not altered after adjustment for total LDL particles. The number of LDL particles may be the better marker for LDL-attributable atherosclerosis because a particle's cholesterol delivered to an atherosclerotic lesion depends mainly on particle number. [27] Accordingly, LAB may be a candidate for one residual risk factor beyond LDLC. A recent 10-year cohort study of 2,436 Japanese residents showed an increase risk of ischemic stroke according to serum LAB elevation [22] despite no association between LDLC and ischemic stroke in this cohort. [4]

There are some enzyme-linked immuno-sorbent assays (ELISA) to measure oxidized LDL. [13] They used antibody against oxidation-dependent one specific antigenic determinant in LDL. [8] In contrast, amongst the apo B-containing lipoproteins, various types of modified LDL, such as malondialdehyde-LDL, 4-hydroxy-2-nonenal (HNE)- LDL [8], and 4-oxo-2nonenal (ONE)-LDL [9] and carbamylated LDL [10] are ligands for LOX-1. Accordingly, measuring total LOX-1 ligands would better reflect the biological activities of apo Bcontaining lipoproteins than measuring one specific antigenic determinant of modified LDL. Therefore, we believe that the ELISA assay for LAB using monoclonal anti-ApoB antibody, which recognizes both ApoB-48 and ApoB-100 as well as recombinant LOX-1, has the ability to evaluate the biological activity of the atherogenic lipoproteins. [12]

Since we used the receptor LOX-1 to detect LAB, LAB is not merely a marker but is considered to be ready to bind to LOX-1 and induce subsequent reactions in vivo including endothelial dysfunction [8] leading to lipid deposition [28] and inflammation [29], smooth muscle migration and proliferation [30], and foam cell formation. [31] Although the evidence of responses to LOX-1 activation have been based on in vitro experiments or animal experiments mostly in rodents, our present and previous studies support that the concept might be applicable to human, showing that high LAB concentration predicted the risk of future events of cardiovascular disease in Japanese cohort [21] and associated with IMT of carotid artery in US Caucasian men. Interestingly, while acute elevation of sLOX-1 on acute coronary syndrome has been reported [32,33], LAB, but not sLOX-1, was primarily associated with future event risk and carotid IMT. This might suggest that LAB concentration is rate limiting in steady state of atherosclerosis in healthy people.

In the present study, serum LAB was not associated with IMT among Japanese. Although LDLC is a risk factor for CAD even in middle-aged Japanese [3,4], absolute risk for CAD in Japanese is very low [34], which is similar to that of decade younger Americans. [35] In fact, in the age group of the present study, aged 40 to 49, average IMT was much lower 
compared with US Caucasian men; and the association between LDL-C per se and IMT was not observed in Japanese in our previous report. [15] Atherogenic effect of LAB may be observed in a population where the absolute risk of CAD due to hypercholesterolemia is relatively high such as in Americans or elderly Japanese. Another possible reason is atherogenic effect of LAB has a threshold level. In other words, the graded relationship between $\mathrm{LAB}$ and atherosclerotic disease may be only observed above a certain level of LAB. In a previous cohort study [21], the relationship between LAB and cardiovascular disease did not show a linear trend, but was discontinuously high in the top quartile and low in the bottom quartile. However, further research should be warranted.

The present study has some limitations. First, the study was cross-sectional, which cannot prove a causal relation. Second, a study in men aged 40-49 may limit generalization to older individuals and women. However, we believe our sample limited age and sex group is suitable to examine an effect of new biomarker on IMT because it may eliminate the inevitable confounding due to aging and sex. Third, 36 out of 297 US Caucasian men and 10 out of 301 Japanese men were prescribed some lipid-lowering agents, which may affect our results. However, statistical adjustment for lipid lowering medication or exclusion for participants who are taking lipid lowering medication did not alter our findings. Finally, we used carotid IMT as subclinical atherosclerosis, which was associated with future risk of $\mathrm{CAD}$ and stroke in previous studies; however, whether the progression of IMT predicts future CVD or not is under intense debate. [36]

In conclusion, serum level of LAB was higher among middle-aged US Caucasian men than Japanese men, and was associated with average IMT among US Caucasian men only. However, reasons for these findings are unclear. Because there are very few clinical and epidemiologic studies concerning LAB and SLOX-1, further studies should be warranted in women, different age or different ethnic groups. LAB may provide a new insight into not only risk stratification but also therapeutic strategy of atherosclerotic disease.

\section{Supplementary Material}

Refer to Web version on PubMed Central for supplementary material.

\section{Acknowledgments}

Source of funding

This research was supported by grants R01 HL68200 and HL071561 from the National Institutes of Health. This research was supported by a Grant-in-Aid for Scientific Research ((B):23390178) by the Japan Society for the Promotion of Science and a Grant-in-Aid for Scientific Research ((A):13307016) by the Japanese Ministry of Education, Culture, Sports, Science and Technology

This study was approved by the Institutional Review Boards of Shiga University Medical Science, Otsu, Japan and University of Pittsburgh, Pittsburgh, US. We sincerely appreciate researchers and co-medical staffs in the ERA JUMP (Electron-Beam Tomography, Risk Factor Assessment Among Japanese and U.S. Men in the Post-World War II Birth Cohort) Study Group.

\section{References}

1. Task Force for the management of dyslipidaemias of the European Society of Cardiology (ESC) and the European Atherosclerosis Society (EAS) ESC Committee for Practice Guidelines 2008-2010 and 2010-2012 Committees. ESC/EAS Guidelines for the management of dyslipidaemias: the Task Force for the management of dyslipidaemias of the European Society of Cardiology (ESC) the European Atherosclerosis Society (EAS). Atherosclerosis. 2011; 217 (Suppl 1):S1-44. [PubMed: 21723445] 
2. Nagasawa SY, Okamura T, Iso H, et al. Relation between serum total cholesterol level and cardiovascular disease stratified by sex and age group: a pooled analysis of 65594 individuals from 10 cohort studies in Japan. J Am Heart Assoc. 2012; 1:e001974. [PubMed: 23316288]

3. Teramoto T, Sasaki J, Ishibashi S, et al. Executive Summary of Japan Atherosclerosis Society (JAS) Guideline for Diagnosis and Prevention of Atherosclerotic Cardiovascular Diseases for Japanese-2012 version. J Atheroscler Thromb. in press.

4. Okamura T, Kokubo Y, Watanabe M, et al. A revised definition of the metabolic syndrome predicts coronary artery disease and ischemic stroke after adjusting for low density lipoprotein cholesterol in a 13-year cohort study of Japanese: the Suita study. Atherosclerosis. 2011; 217:201-6.4. [PubMed: 21481396]

5. Mudd JO, Borlaug BA, Johnston PV, et al. Beyond low-density lipoprotein cholesterol: defining the role of low-density lipoprotein heterogeneity in coronary artery disease. J Am Coll Cardiol. 2007; 50:1735-41. [PubMed: 17964036]

6. Stocker R, Keaney JF Jr. Role of oxidative modifications in atherosclerosis. Physiol Rev. 2004; 84:1381-478.7. [PubMed: 15383655]

7. Sawamura T, Kume N, Aoyama T, et al. An endothelial receptor for oxidized low-density lipoprotein. Nature. 1997; 386:73-77. [PubMed: 9052782]

8. Sawamura T, Kakino A, Fujita Y. LOX-1: a multiligand receptor at the crossroads of response to danger signals. Curr Opin Lipidol. 2012; 23:439-459. [PubMed: 22777292]

9. Shibata T, Shimozu Y, Wakita C, et al. Lipid peroxidation modification of protein generates Nepsilon-(4-oxononanoyl) lysine as a pro-inflammatory ligand. J Biol Chem. 2011; 286:19943-57. [PubMed: 21471194]

10. Kumano-Kuramochi M, Shimozu Y, Wakita C, et al. Identification of 4-hydroxy-2-nonenalhistidine adducts that serve as ligands for human lectin-like oxidized LDL receptor-1. Biochem J. 2012; 44:171-80. [PubMed: 22077443]

11. Apostolov EO, Shah SV, Ray D, Basnakian AG. Scavenger receptors of endothelial cells mediate the uptake and cellular proatherogenic effects of carbamylated LDL. Arterioscler Thromb Vasc Biol. 2009; 29:1622-1630. [PubMed: 19696406]

12. Sato Y, Nishimichi N, Nakano A, et al. Determination of LOX-1 ligand activity in mouse plasma with a chicken monoclonal antibody for ApoB. Atherosclerosis. 2008; 200:303-309. [PubMed: 18346743]

13. Itabe H, Ueda M. Measurement of plasma oxidized low-density lipoprotein and its clinical implications. J Atheroscler Thromb. 2007; 14:1-11. [PubMed: 17332686]

14. Yamazaki K, Bujo H, Taira K, et al. Increased circulating malondialdehyde-modified LDL in the patients with familial combined hyperlipidemia and its relation with the hepatic lipase activity. Atherosclerosis. 2004; 172:181-7. [PubMed: 14709374]

15. Sekikawa A, Ueshima H, Kadowaki T, et al. Less subclinical atherosclerosis in Japanese men in Japan than in White men in the United States in the post-World War II birth cohort. Am J Epidemiol. 2007; 165:617-24. [PubMed: 17244636]

16. Sekikawa A, Ueshima H, Sutton-Tyrrell K, et al. Intima-media thickness of the carotid artery and the distribution of lipoprotein subclasses in men aged 40 to 49 years between whites in the United States and the Japanese in Japan for the ERA JUMP study. Metabolism. 2008; 57:177-82. [PubMed: 18191046]

17. Sekikawa A, Curb JD, Ueshima H, et al. Marine-derived n-3 fatty acids and atherosclerosis in Japanese, Japanese-American, and white men: a cross-sectional study. J Am Coll Cardiol. 2008; 52:417-24. [PubMed: 18672160]

18. Okamura T, Sekikawa A, Kadowaki T, et al. Cholesteryl ester transfer protein, coronary calcium, and intima-media thickness of the carotid artery in middle-age Japanese men. Am J Cardiol. 2009; 104:818-22. [PubMed: 19733717]

19. Friedewald W, Levy R, Fredrickson D. Estimation of the concentration of low density lipoprotein cholesterol in plasma without use of the ultracentrifuge. Clin Chem. 1972; 18:499-502. [PubMed: 4337382]

20. Murase T, Kume N, Kataoka H, et al. Identification of soluble forms of lectin-like oxidized LDL receptor-1. Arterioscler Thromb Vasc Biol. 2000; 20:715-20. [PubMed: 10712396] 
21. Inoue N, Okamura T, Kokubo Y, et al. LOX index, a novel predictive biochemical marker for coronary heart disease and stroke. Clin Chem. 2010; 56:550-8. [PubMed: 20093560]

22. Thompson T, Sutton-Tyrrell K, Wildman R. Continuous quality assessment programs can improve carotid duplex scan quality. Journal of Vascular Technology. 2001; 25:33-39.

23. Liu ML, Ylitalo K, Salonen R, Salonen JT, Taskinen MR. Circulating oxidized low-density lipoprotein and its association with carotid intima-media thickness in asymptomatic members of familial combined hyperlipidemia families. Arterioscler Thromb Vasc Biol. 2004; 24:1492-7. [PubMed: 15205217]

24. Meisinger C, Baumert J, Khuseyinova N, Loewel H, Koenig W. Plasma oxidized low-density lipoprotein, a strong predictor for acute coronary heart disease events in apparently healthy, middle-aged men from the general population. Circulation. 2005; 112:651-7. [PubMed: 16043640]

25. Koenig W, Karakas M, Zierer A, et al. Oxidized LDL and the risk of coronary heart disease: results from the MONICA/KORA Augsburg Study. Clin Chem. 2011; 57:1196-200. [PubMed: 21697499]

26. Wu T, Willett WC, Rifai N, Shai I, Manson JE, Rimm EB. Is plasma oxidized low-density lipoprotein measured with the widely used antibody 4E6 an independent predictor of coronary heart disease among U.S. men and women? J Am Coll Cardiol. 2006; 48:973-9. [PubMed: 16949489]

27. Tabas KJ, Williams, Boren J. Subendothelial lipoprotein retention as the initiating process in atherosclerosis: update and therapeutic implication. Circulation. 2007; 116 :1832-1844. [PubMed: 17938300]

28. Ishigaki Y, Katagiri H, Gao J, et al. Impact of plasma oxidized low-density lipoprotein removal on atherosclerosis. Circulation. 2008; 118:75-83. [PubMed: 18559699]

29. Honjo M, Nakamura K, Yamashiro K, et al. Lectin-like oxidized LDL receptor-1 is a cell-adhesion molecule involved in endotoxin-induced inflammation. Proc Natl Acad Sci U S A. 2003; 100:1274-1279. [PubMed: 12538855]

30. Hinagata J, Kakutani M, Fujii T, et al. Oxidized LDL receptor LOX-1 is involved in neointimal hyperplasia after balloon arterial injury in a rat model. Cardiovasc Res. 2006; 69:263-271. [PubMed: 16183045]

31. Li L, Sawamura T, Renier G. Glucose enhances human macrophage LOX-1 expression: role for LOX-1 in glucose-induced macrophage foam cell formation. Circ Res. 2004; 94:892-901. [PubMed: 15001526]

32. Hayashida K, Kume N, Murase T, et al. Serum soluble lectin-like oxidized low-density lipoprotein receptor-1 levels are elevated in acute coronary syndrome: a novel marker for early diagnosis. Circulation. 2005; 112:812-8. [PubMed: 16061745]

33. Kobayashi N, Hata N, Kume N, et al. Matrix metalloproteinase- 9 for the earliest stage acute coronary syndrome. Circ J. 2011; 75:2853-61. [PubMed: 21963570]

34. NIPPON DATA80 Research Group. Risk assessment chart for death from cardiovascular disease based on a 19-year follow-up study of a Japanese representative population. Circ J. 2006; 70:1249-55. [PubMed: 16998254]

35. Saito I, Folsom AR, Aono H, Ozawa H, Ikebe T, Yamashita T. Comparison of fatal coronary heart disease occurrence based on population surveys in Japan and the USA. Int J Epidemiol. 2000; 29:837-44. [PubMed: 11034966]

36. Lorenz MW, Polak JF, Kavousi M, et al. Carotid intima-media thickness progression to predict cardiovascular events in the general population (the PROG-IMT collaborative project): a metaanalysis of individual participant data. Lancet. 2012; 379:2053-62. [PubMed: 22541275] 


\section{Highlight}

- We recruited community-dwelling 297 US Caucasian and 310 Japanese men, aged 40 to 49 years.

- The serum level of LOX-1 ligand containing ApoB (LAB) was measured.

- LAB was significantly higher in US men than in Japanese men.

- LAB was significantly associated with IMT after adjustment for total LDL particles in US Caucasian men.

- There was no significant relationship between LAB and IMT in Japanese men. 


\section{Table 1}

Comparison of Basic Characteristics between 297 US Caucasian men aged 40-49 years in Allegheny County, Pennsylvania and 310 Japanese men aged 40-49 years in Kusatsu City, Shiga, Japan ${ }^{\dagger}$

\begin{tabular}{lccc}
\hline Population & Caucasian men & Japanese men & P-values \\
$\mathbf{N}$ & $\mathbf{2 9 7}$ & $\mathbf{3 1 0}$ & \\
\hline Age, year & $45.0(2.8)$ & $45.1(2.8)$ & 0.735 \\
LAB, $\mu \mathrm{g} / \mathrm{L}^{\neq}$ & $1321(936,1730)$ & $940(688,1259)$ & $\mathrm{P}<0.001$ \\
sLOX-1, ng/L ${ }^{\neq}$ & $520(345,763)$ & $328(157,832)$ & $\mathrm{P}<0.001$ \\
BMI, kg/m2 & $28.0(4.4)$ & $23.7(3.1)$ & $\mathrm{P}<0.001$ \\
LDL cholesterol, mmol/L & $3.50(0.87)$ & $3.42(0.93)$ & 0.312 \\
HDL cholesterol, mmol/L & $1.23(0.33)$ & $1.40(0.35)$ & $\mathrm{P}<0.001$ \\
Total LDL particles, nmol/L & $1461(343)$ & $1473(407)$ & 0.685 \\
Triglyceride, mmol/L ${ }^{\neq}$ & $1.46(1.04,2.10)$ & $1.53(1.16,2.05)$ & 0.159 \\
Hypertension, \% & 15.8 & 25.8 & 0.003 \\
Systolic blood pressure, mmHg & $123(11)$ & $125(16)$ & 0.005 \\
Diastolic blood pressure, mmHg & $73(9)$ & $76(12)$ & $\mathrm{P}<0.001$ \\
Anti-hypertensive medication, \% & 9.1 & 5.2 & 0.081 \\
Diabetes, \% & 3.4 & 6.1 & 0.129 \\
CRP, g/L $\neq$ & 0.39 & 1.00 & $\mathrm{P}<0.001$ \\
Current smoking, \% & 8.1 & 49.0 & $\mathrm{P}<0.001$ \\
Current drinker, \% & 43.8 & 67.1 & $\mathrm{P}<0.001$ \\
Lipid lowering medication, \% & 12.1 & 3.2 & $\mathrm{P}<0.001$ \\
Average IMT, mm & $0.678(0.103)$ & $0.614(0.071)$ & $\mathrm{P}<0.001$ \\
\hline
\end{tabular}

LAB means "LOX-1 legands containing apolipoprotein B". sLOX-1 means "soluble form of lecitin-like oxidized LDL receptor 1".

HDL means "high-density lipoprotein". LDL means "low-density lipoprotein". IMT means "intima-media thickness".

Hypertension was defined as systolic blood pressure $\geq 140 \mathrm{~mm} \mathrm{Hg}$, diastolic blood pressure $290 \mathrm{~mm}$ Hg, or hypertensive medication.

Diabetes was defined as fasting glucose $\geq 7.0 \mathrm{mmol} / \mathrm{L}$ ( $\geq 126 \mathrm{mg} / \mathrm{dl}$ or diabetes medication).

CRP means C-reactive protein.

${ }^{\dagger}$ Values are expressed as mean (standard deviation) for continuous variables or as percentage for categorical variables.

Fedian (interquartile range). 
告 


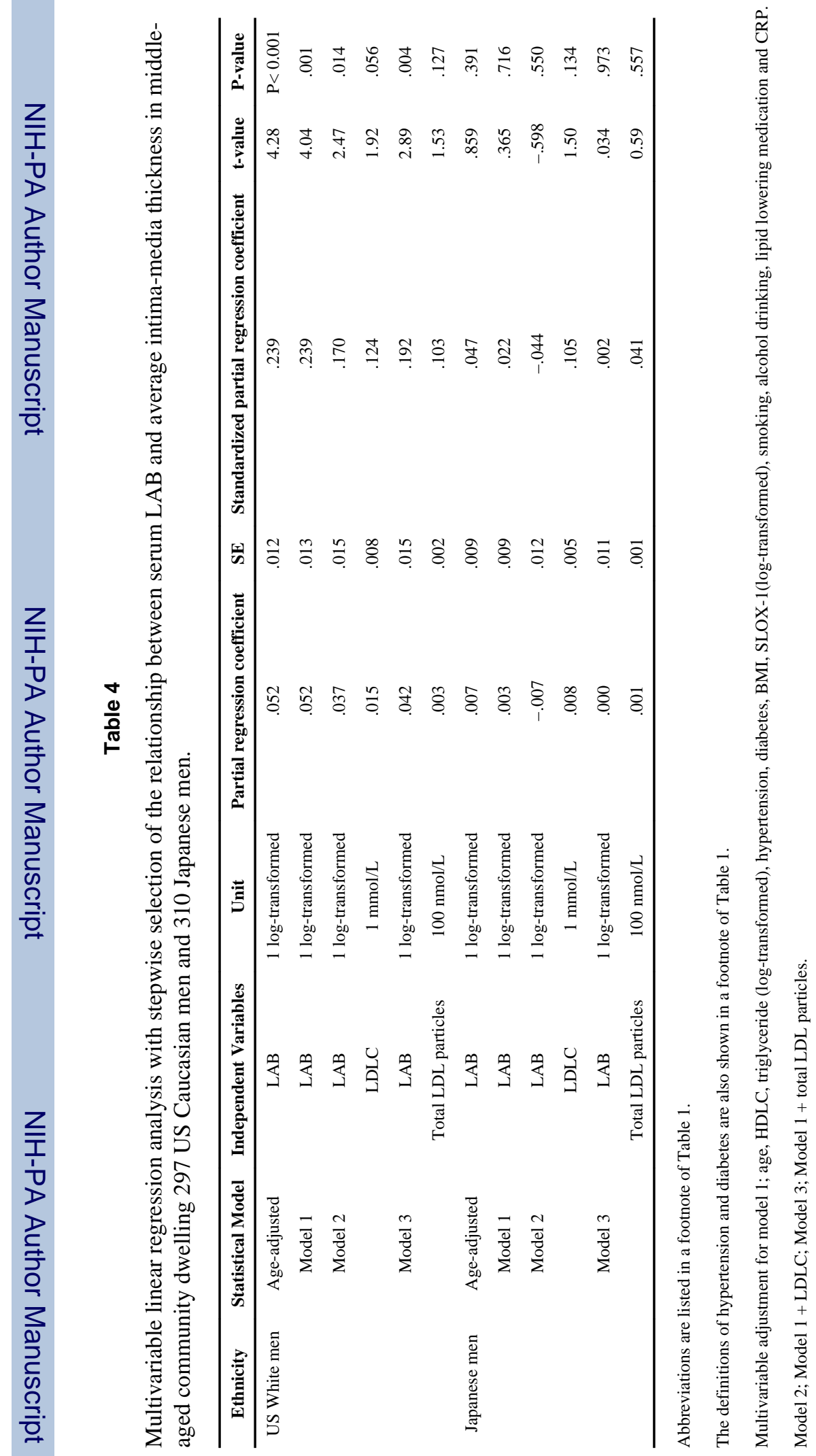

Atherosclerosis. Author manuscript; available in PMC 2013 July 01. 